\title{
A espacialização do câncer mamário e uterino em Porto Velho, RO
}

\author{
The spatialization of mammary and uterine cancer in \\ Porto Velho, RO
}

\section{La espacialización del cáncer mamario y uterino en Porto Velho, RO}

\author{
Sheila Castro dos Santos \\ https:/ / orcid.org/0000-0003-1704-5742 \\ sheila1705@gmail.com
}

Universidade Estadual de Londrina, UEL, Londrina, PR

Geisa Chaves do Nascimento dos Santos

https:/ / orcid.org/0000-0002-1255-1147

geisasof4@hotmail.com

Universidade Federal de Rondônia, UNIR, Porto Velho, RO

\begin{abstract}
Resumo: Este artigo sob a temática da Geografia a Saúde apresenta e discute a incidência e espacialização do câncer em Porto Velho, de 2012 a 2016, com dados fornecidos pelo Hospital de Base Dr. Ary Pinheiro, e quais medidas são efetivadas pelo poder público para o combate da endemia. Porto Velho mostrou números altos de câncer, tanto de mama quanto de útero, principalmente por falta de informação. Os índices mais altos estão nos bairros que concentram as mulheres trabalhadoras defrontadas com a escolha entre ganhar o salário para sua manutenção e a prevenção básica de sua saúde. As concepções da Geografia da Saúde neste trabalho envolveram a análise não só das pessoas envolvidas direta ou indiretamente, como é o caso dos pacientes e dos atendentes, mas também das políticas públicas de saúde. Também foi evidente o descompromisso com o preenchimento dos dados de anamnese, dificultando as pesquisas em Geografia da Saúde.
\end{abstract}

Palavras-chave: Prevenção do câncer, Saúde da Mulher, Políticas Públicas de Saúde, Geografia da Saúde.

\begin{abstract}
This paper of Health Geography presents and discusses the incidence and spatialization of cancer in Porto Velho, from 2012 to 2016, with data provided by the Hospital de Base Dr. Ary Pinheiro, and which measures are effective to combat this endemic disease. Porto Velho showed high numbers of cancer cases, both breast and uterus, mainly due to a lack of information. The highest rates are in neighborhoods that concentrate on working women faced with the choice between earning the salary for their maintenance and the basic prevention of their health. The conceptions of Health Geography in this work involved the analysis not only of the people directly
\end{abstract}


or indirectly involved, as patients and attendants but also of health public policies. It was also evident the disengagement with the completion of anamnesis data, hindering research in Health Geography.

Keywords: Cancer Prevention, Health of Women, Health Public Policies, Health Geography.

Resumen: Este artículo bajo el tema de Geografía de la Salud presenta y analiza la incidencia y espacialización del cáncer en Porto Velho, de 2012 a 2016, con datos proporcionados por el Hospital de Base Dr. Ary Pinheiro, y qué medidas el poder público efectiva combater la endemía. Porto Velho mostró un alto número de cáncer, tanto de mama como de útero, principalmente debido a la falta de información. Las tasas más altas se encuentran en los barrios que concentran a las mujeres trabajadoras que se enfrentan a la elección entre ganar el salário por su manutención y la prevención básica de su salud. Las concepciones de la Geografía de la Salud en este trabajo involucraron el análisis no sólo de las personas directa o indirectamente involucradas, como los pacientes y asistentes, sino también de las políticas públicas de salud. También fue evidente la desvinculación con la finalización de los datos de anamnesis, obstaculizando la investigación en Geografía de la Salud.

Palabras clave: Prevención de cáncer, Salud de la Mujer, Políticas Pública de Salud, Geografía de la Salud.

\section{INTRODUÇÃO}

De acordo com estimativas do Instituto Nacional de Câncer (INCA) para os biênios 2018-2019 estima-se a ocorrência de 600 mil casos de câncer no Brasil. Essas estimativas refletem o perfil do câncer em um país que possui as neoplasias de mama e útero como as que mais acometem mulheres no Brasil.

Na construção epidemiológica do câncer elaborada por Sodré (1984, p. 18), percebeu-se que as espacializações das endemias estavam vinculadas às questões referentes ao processo saúde/doença desde o cerne das preocupações humanas. "O ser humano, desde o início da sua existência, está suscetível a uma grande variedade de moléstias causadas pelos mais diversos fatores, sejam eles físicos, sociais, ambientais ou psíquicos e, desde os tempos mais remotos, a busca pelo tratamento, cura e prevenção dessas, permeiam as ações humanas". Muitas dessas doenças já foram erradicadas, ao passo que outras surgiram em outros tempos. Atualmente, "as neoplasias malignas ou cânceres se destacam nos debates referentes à saúde por todo o mundo".

A epidemiologia do câncer chama a atenção pela forma de como essa endemia vem incidindo no espaço geográfico. Para Lacaz, Baruzzi e Siqueira Junior (1972), a neoplasia maligna (câncer) é um novo capítulo para uma análise da geografia. Os fatores que contribuem para a distribuição do câncer no espaço são vários, os mais importantes, segundo Carvalho (1972, p. 489) “são fatores como idade, nível sócio econômico, hábitos e fatores culturais".

Em sua reflexão sobre o espaço Santos (2006) indica a relação direta entre tempo e espaço, por meio de técnicas torna-se possível a compreensão dinâmica do processo 
da construção social do espaço geográfico advindo das transformações decorrentes das metamorfoses ambientais, sociais e comportamentais. Portanto, mudanças espaciais se refletem na vida social dos grupos envolvidos. Para Santos (2006) o espaço é estático, não muda sem a intervenção homem, produto do espaço no qual se insere, e no qual produz as mudanças e vive suas consequências. Santos (1998) afirma ainda que o lugar atribui a cada elemento constituinte do espaço um valor particular. Completa Barcellos, Sabroza, Peiter e Rojas (2002) que o espaço, produto e produtor, enquanto lugar onde vivenciamos as nossas transformações, adquire características diversas de acordo com a região, hábitos e cultura.

Foi através da abordagem e da análise social do espaço que Santos (1998) contribuiu efetivamente para os trabalhos de Geografia da Saúde e sua distribuição desigual, ou seja, as formas como os acontecimentos se disseminam de acordo com o seu tempo e localização geográfica:

O espaço possui duas dimensões uma histórica e outra temporal, assim, o espaço deve ser considerado como um conjunto de relações realizadas através de testemunho de uma história escrita por processos do passado e do presente e por uma estrutura representada por relações sociais que estão acontecendo diante de nossos olhos e que se manifestam através de processos e funções. O espaço é, então, um verdadeiro campo de forças. (Santos, 1998, p. 79).

Logo, o espaço é fruto das relações históricas e temporais. A partir deste princípio não há como pensar o espaço sem relacioná-lo as transformações, onde as combinações entre técnica, tempo e história são formas de consolidar os acontecimentos históricos vivenciados no espaço.

\section{GEOGRAFIA DA SAÚDE}

Com a criação das Faculdades de Medicina no país, em 1808, surgiram diversos trabalhos que abordavam questões referentes à geografia das doenças ou patologia geográfica das várias regiões brasileiras.

Em 1972 Lacaz, Baruzzi e Siqueira Junior publicaram o livro Introdução à Geografia Médica no Brasil, que apesar de não conseguir cumprir seu objetivo de integrar tais conhecimentos, tem contribuído para o resgate histórico das discussões entre geografia e saúde. Pode-se dizer, que esse período foi marcado por uma Geografia Médica atrelada aos interesses do governo e da classe dominante, não havendo uma análise mais crítica que relacionasse esses estudos aos fatores socioeconômicos e culturais.

Retornando como conhecimento estratégico durante a Segunda Guerra mundial, a Geografia Médica tem seus estudos atrelados a União Geográfica Internacional. Em 1949, a criação da Comissão de Geografia Médica da União Geográfica Internacional - UGI - possibilitou o avanço nos estudos referentes a doenças e geografia. Em reunião ocorrida em 1976, em Moscou, foi solicitado a mudança da denominação de Geografia Médica para Geografia da Saúde. Para Junqueira (2009, p.5) isso ocorreu dado o crescimento da temática e do tipo de abordagem "considerada mais abrangente por relacionar a qualidade de vida, a 
educação, à moradia, o saneamento básico, infraestrutura em saúde e outros com a saúde das populações", contudo, segundo Rojas (1998), a denominação Geografia Médica ainda é utilizada em alguns países.

Como afirma Teixeira (2009), o homem sofre com as transformações no espaço, muitas vezes ocasionadas por ele próprio, com as divergências sociais, econômicas, culturais e ambientais, que fazem com que as doenças incidam no espaço de forma diferenciada. No cotidiano a Geografia da Saúde estuda a distribuição desigual das doenças e a forma da aplicação das políticas públicas voltadas a prevenção e/ou tratamento da enfermidade, entre elas o câncer.

Segundo Peiter (2005), com a interferência da corrente neopositivista, a Geografia da Saúde procurou identificar regularidades nos modos de propagação de doenças no tempo e no espaço, ganhando enorme força na década de 1980, com os avanços da informática e as novas ferramentas de softwares de estatística, de mapeamento digital e Sistemas de Informação Geográfica. Novas técnicas de geoprocessamento têm contribuído para avanços no campo da Geografia da Saúde e no estudo de diferentes fatores que intervém na propagação de doenças.

Graças à necessidade de se ter uma melhor interpretação dos fenômenos da saúde, a Geografia da Saúde vem sendo cada vez mais relacionada com os processos de globalização, o que na visão de Rojas (1998) vem resultando em novo paradigma no contexto da Geografia e Saúde onde a saúde se relaciona diretamente à qualidade de vida, índice de desenvolvimento humano e acesso à infraestrutura, como transportes, educação e equipamentos urbanos, dentre outros.

\section{CONTEXTUALIZAÇÃO BREVILOQUENTE DO CÂNCER DE ÚTERO E MAMA}

Já no século XX se alertava para a dificuldade de conhecer melhor o câncer devido à inexistência de dados epidemiológicos. Um dos primeiros trabalhos médicos tratando a doença como problema coletivo no Brasil, foi publicado por Azevedo Sodré (1984), denominado A frequência do câncer no Brasil. Também nesse período, se intensificaram as preocupações com o câncer na sociedade brasileira. Recentemente seu progressivo impacto epidemiológico e a necessidade de maior cobertura das ações, visando seu controle, principalmente pelo Sistema Único de Saúde (SUS), têm colocado a doença no centro das discussões sobre medicina e saúde pública no Brasil. Nesse contexto, é importante analisar a historicidade dessas preocupações sociais e seus aspectos menos evidentes. O estatuto atual do câncer se relaciona diretamente ao incremento de sua incidência e à prevalência nas diversas regiões do país, bem como às tecnologias, às iniciativas médicas e às políticas públicas surgidas para o seu controle.

De acordo com o INCA, mais de 200 doenças recebem o nome genérico de câncer. Alguns tipos de câncer começam devido ao crescimento anormal das células. Contudo, segundo Lima e Zeferino (2008) o câncer resulta da interação entre a suscetibilidade genética e fatores ou condições relacionadas ao modo de vida e ao ambiente em que vivem os indivíduos. Liu e Robins (2006) destaca que a incidência de determinados cânceres também 
aumenta com exposição a agentes carcinogênicos como fumaça de cigarro, compostos químicos e radiação; ainda, chama a atenção, outros fatores ambientais também devem ter papel importante, já que a prevalência de certos tipos de câncer varia de acordo com as políticas públicas de algumas regiões. Segundo Fonseca (2007) esta teoria contrapõe a visão de uni casualidade e abre caminho para as teorias multicausais do câncer.

Segundo o INCA, os fatores externos estão relacionados às questões ambientais, a hábitos de vida, e as questões culturais, econômicos dentre outros; os internos são geneticamente pré determinados. Estando ligados a capacidade do organismo de se defender das agressões externas, esses fatores podem agir de várias formas, aumentando a probabilidade de transformações malignas nas células normais - "a medida que a sua alimentação é melhor balanceada e que a medicina preventiva ocupa o lugar que deve, o gênero humano aumenta gradativamente a média de vida" (Marsillac \& Merched, 1954, p. 42). Mesmo sem poder afirmar com exatidão as causas da incidência de câncer, é possível inferir que os fatores internos e externos estão correlacionados.

De acordo com a Fundação Oswaldo Cruz ([FioCruz] 2018) o câncer é uma doença conhecida há longa data, os relatos sobre sua existência remontam à Antiguidade, e sempre são relacionados a sofrimento e morte. No Brasil, o câncer era considerado uma doença de pequena incidência, mas incurável. Provavelmente o desconhecimento em torno do problema e a falta de técnicas para diagnósticos preciso causaram essa impressão. A situação financeira não favorecia o diagnóstico preciso da doença, logo, só havia diagnóstico de câncer nos membros da elite que realizavam consultas médicas, enquanto grande parte da população não tinha acesso aos hospitais. A primeira iniciativa para o controle do câncer no Brasil foi no início do século XX, direcionada ao diagnóstico e tratamento. Nesta época havia muita escassez de informação sobre a doença o que impossibilitava falar em prevenção.

Conforme Ministério da saúde, o crescente número de pessoas diagnosticadas com câncer no Brasil e no mundo é decorrente do aumento da expectativa de vida e, segundo o INCA (2018), as maiores taxas de incidência ocorrem em países desenvolvidos. No Brasil, de acordo com o INCA (2009) são esperados 17.540 novos casos de câncer de mama e útero, e a região de maior incidência será o Norte do país; nas regiões Sul e Sudeste a incidência é predominantemente dos canceres de mama feminina, pulmão e intestino; no Centro Oeste os canceres de estômago e útero são os mais prevalentes

O câncer hoje é, portanto, encarado como um problema de saúde pública. Os elevados índices de incidência e mortalidade por câncer do colo do útero e da mama no Brasil justificam a implantação de estratégias efetivas de controle dessas doenças que incluam ações de promoção à saúde, prevenção e detecção precoce, tratamento e de cuidados paliativos, quando esses se fizerem necessários. A importância epidemiológica do câncer no Brasil e sua magnitude social, as condições de acesso da população brasileira à atenção oncológica, os custos cada vez mais elevados na alta complexidade, refletem a necessidade de estruturar uma rede de serviços regionalizada e hierarquizada que garanta atenção integral à população. 
Com relação à detecção precoce, a maior parte dessas ações ocorre na Atenção Básica. Tanto as ações de rastreamento, que consistem em realizar sistematicamente testes ou exames em pessoas sadias, quanto as ações de diagnóstico precoce, que consistem em captar precocemente alguém que já tem sintomas ou alterações no exame físico, devem fazer parte da rotina das equipes. Também cabe destacar o papel da Atenção Básica no que tange aos cuidados paliativos, uma vez que o tratamento dos cânceres nem sempre é curativo. Mesmo diante de todo o aporte tecnológico atual, existem falhas terapêuticas, que ocorrem com mais frequência quando o quadro da doença está mais avançado. Nesse sentido, muitas vezes o tratamento visa diminuir o tumor existente e aumentar a sobrevida e a qualidade de vida, uma vez que a usuária passará a conviver com a doença por um período variado.

As atividades a serem realizadas para promover a saúde incluem a boa nutrição, $\mathrm{o}$ atendimento às necessidades afetivas, educação sexual, orientação pré-nupcial e parental, boas condições habitacionais, trabalho e lazer, além de exames periódicos e educação para a saúde. Em suma, inclui-se o período pré-sintomático das doenças, viabilizando o surgimento do movimento de Medicina Preventiva dentro dos sistemas de saúde pública.

As ações da Atenção Básica podem ser diversas no controle dos cânceres do colo do útero e da mama. Vão desde cadastro e identificação da população prioritária ao acompanhamento das usuárias em cuidados paliativos.

Segundo o INCA (2009), o câncer de colo uterino, também chamado de cervical, é caracterizado pela replicação desordenada do epitélio de revestimento da cavidade uterina. É uma doença de evolução lenta que passa por fases detectáveis e curáveis. Estima-se que o tempo entre a lesão inicial e fase clínica leva entre 10 a 15 anos.

As alterações das células que podem desencadear o câncer são descobertas facilmente no exame preventivo de colpocitologia oncótica cervical (também conhecido como Papanicolau), por isso a importância de sua realização periódica. Sua incidência evidencia-se na faixa etária de 20 a 29 anos e o risco aumenta rapidamente até atingir seu pico, geralmente na faixa etária de 45 a 49 anos. Esse é o câncer que apresenta maior potencial de prevenção e cura quando diagnosticado precocemente (INCA, 2009). Segundo Gallo, Guerra e Mendonça (2005), este tipo de câncer representa um grave problema de saúde pública nos países da América Latina, considerada como uma das regiões de maior incidência no mundo (INCA, 2017). As infecções oncogênicas são causadas, em geral, pelo Vírus Papiloma Humano (HPV 16 e 18).

Embora o câncer cervical seja uma patologia com grande potencial de prevenção e cura, estima-se que, nos países em desenvolvimento, apenas $49 \%$ das mulheres que o adquirem sobrevivem. Nos países desenvolvidos, por outro lado, segundo Moreno, Santos e Pereira (2009), o diagnóstico é realizado precocemente, a cura chega a quase $70 \%$.

Sobre o câncer de mama, também um grande problema de saúde pública mundial, e o mais temido pelas mulheres devido a sua alta frequência e efeitos psicológicos, uma vez que representa a principal causa de morte em mulheres a partir dos 40 anos. Silva e Silva (2005), evidenciam que o processo de envelhecimento implica em modificações celulares e moleculares que tornam o idoso mais vulnerável ao surgimento de neoplasias. Segundo 
dados do Ministério da Saúde (Brasil, 2006), mulheres que engravidam com mais de 30 anos de idade são mais propensas a desenvolver câncer de mama quando comparadas a mulheres que tiveram seus filhos mais jovens. Além da idade ser um dos fatores de maior relevância na incidência do câncer de mama, os anticoncepcionais orais, a menopausa tardia e a terapia de reposição hormonal estão entre os fatores prevalentes em relação ao desenvolvimento de câncer de mama.

Estudos prospectivos de Engel, Kerr, Schlesinger, Sauer e Hölzel (2004) e Ganz et al. (2004), avaliam a perda de qualidade de vida das mulheres submetidas a mastectómica, com piora não só na imagem corporal, mas também na vida sexual, limitação no trabalho e até mesmo mudanças nos hábitos de vida. Mesmo quando o tratamento permite a preservação da mama, ocorrendo apenas a retirada do tumor, observa-se que sua indicação causa medo e crises nos doentes. Segundo Gomes, Skaba e Vieira (2002, p. 201) "no imaginário social a mama costuma ser associada a atos prazerosos como amamentar, seduzir e acariciar, não combinando com a ideia de ser objeto de uma intervenção dolorosa, ainda que necessária". A mama não corresponde apenas ao aspecto físico, ela é vista como um símbolo de feminilidade, possuindo um significado afetivo e psicológico. A doença em si traz um estigma muito forte para a mulher.

Em todo o mundo o câncer de mama é o segundo tipo de câncer mais frequente entre as mulheres e, a cada ano, cerca de $22 \%$ dos casos novos de câncer detectados em mulheres são de mama. Através do autoexame o sinal de alarme é o aparecimento de nódulo ou caroço palpável na mama ou na axila, sobretudo quando não desaparece durante o ciclo menstrual e não muda de local quando apalpado acompanhado ou não de dor. Segundo o INCA (2009), o estado avançado do tumor é o que mais dificulta o tratamento.

É o segundo tumor mais comum entre as mulheres, após o de colo de útero. Apesar de serem considerados tumores de relativamente bom prognóstico, se detectados e tratados oportunamente, os diagnósticos ainda ocorrem em estágios avançados e as taxas de mortalidade por câncer de mama e de colo de útero continuam elevadas no Brasil.

\section{ESPACIALIZAÇÃO DO CANCER NA CIDADE DE PORTO VELHO}

Como o câncer está presente em várias partes do mundo, afetando diversas vidas em diferentes classes sociais, viu-se a importância de entender a espacialização dessa endemia na cidade de Porto Velho por meio da ciência geográfica. Para tal foram elaborados questionamentos norteadores da pesquisa: como se dá a espacialização do câncer de útero e mama em mulheres de Porto Velho? Qual o perfil sócio demográfico das pacientes? Quais as políticas públicas voltadas para prevenção e tratamento da doença?

Esse estudo de cunho geográfico, descritivo, foi realizado por meio de dados de 838 mulheres que realizaram exames para determinar se tinham sido acometidas pela enfermidade, destas 656 foram diagnosticadas com câncer de mama e útero nos anos de $2012 \mathrm{a}$ 2016 registrados no Hospital de Base Dr. Ary Tupinambá Penna Pinheiro. Empregou-se o método do materialismo dialético, um método de interpretação da realidade no qual 
deve-se primeiramente entender como se dá a unidade de opostos, que no caso deste trabalho colocou-se como a doença versus a saúde.

O espaço de Porto Velho vem passando por diversas metamorfoses demográficas desde sua criação, ocupando a margem direita do rio Madeira, no limite dos estados do Acre e Amazonas. De acordo com o IBGE ${ }^{1}$ sua população é estimada em 519.531 habitantes distribuídos numa área de 34.068,50 km² (Fig. 1).

Figura 1: Município de Porto Velho, RO.

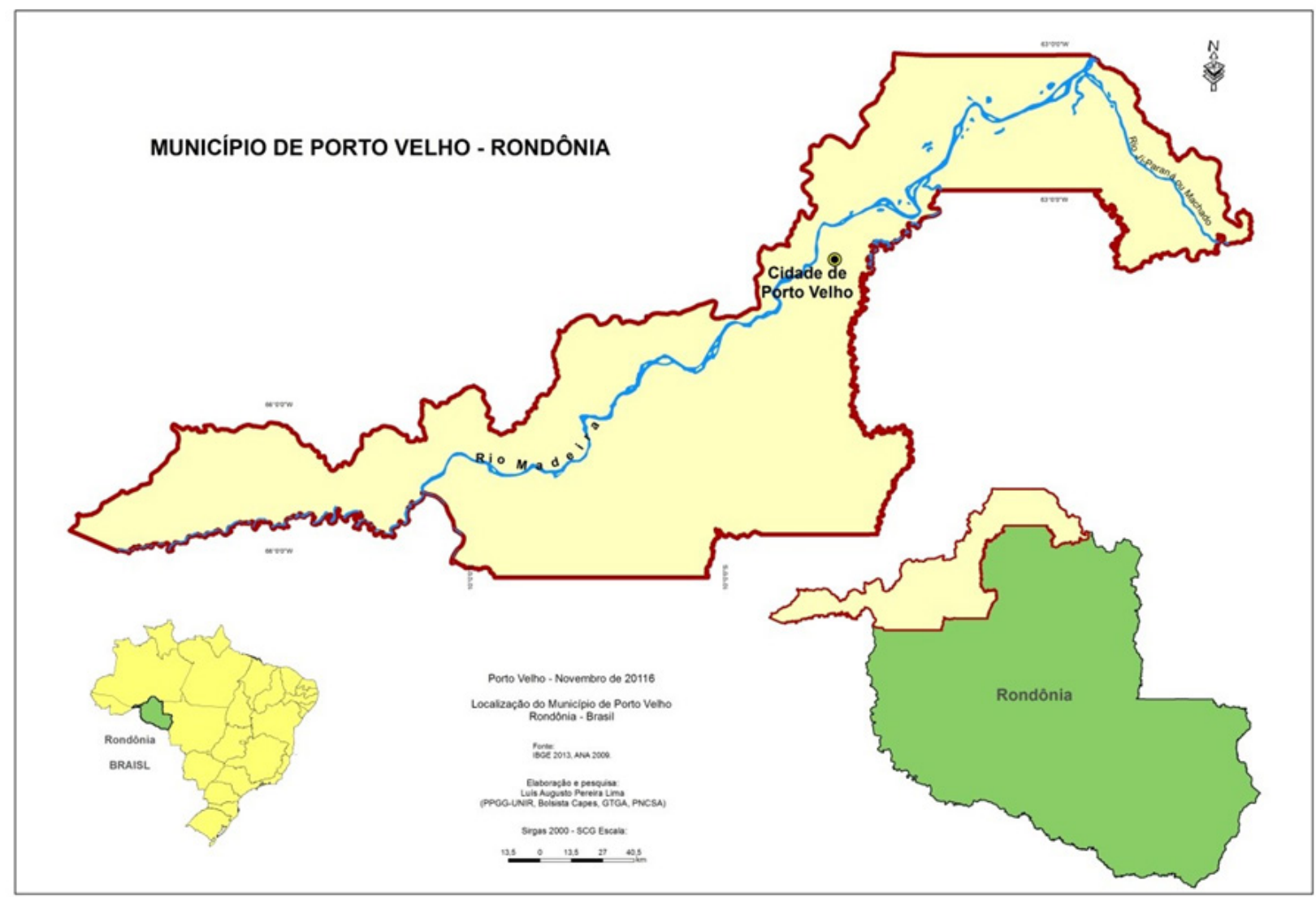

Fonte: Lima e Michalski (2016, p.3).

Desde a década de 1980 a cidade recebe pessoas vindas de diversas regiões do país para trabalhar em vários impulsos econômicos, o mais recente a construção das duas usinas hidrelétricas, de Santo Antônio e Jirau. O crescente aumento populacional fez com que aumentassem as demandas pelos serviços públicos, acentuando a já insuficiente estrutura pública de saúde. Embora existam 44 Unidades Básica de Saúde, três Unidades de Pronto Atendimento (UPA) e quatro veículos no Serviço de Atendimento Móvel as Urgências (SAMU), além dos Hospitais que prestam atendimento de média e alta complexibilidade (João Paulo II e Hospital de Base), os problemas passaram a incidir com mais frequência diante do aumento das demandas. 
Segundo Barcellos, Sabroza, Peiter e Rojas (2002), lugares resultam do acúmulo de situações históricas, ambientais e sociais que geram condições particulares para ocorrência de doenças e, diante de alguns dos fatores como a falta de recursos públicos os problemas de saúde são vistos com frequências como resultantes da falta de políticas públicas comprometidas com a comunidade.

Em 1983, foi criado o Hospital de Base Dr. Ary Tupinambá Penna Pinheiro (HBAP), o qual foi encarregado de realizar o tratamento de pessoas com câncer, sendo até então, o único do Estado de Rondônia. Construído na capital para atender Rondônia, ultrapassou sua função, pois além de atender os cinquenta e dois municípios do estado, realiza atendimento para os munícipes do Acre, Amazonas e Mato Grosso.

O Hospital de Base, o único do Estado de Rondônia encarregado de realizar o tratamento de pessoas com câncer, foi inaugurado em 12 de janeiro de 1983. O Núcleo Hospitalar de Epidemiologia (NHE) foi implantado em 2005 para dar suporte direto à Direção Geral do hospital, segundo critérios pré-estabelecidos pela Secretaria de Vigilância em Saúde do Ministério de Saúde (SVS/MS). Em 2007, o hospital contou com a implantação das Unidades de Assistência de Alta Complexidade (UNACON), que possuem condições técnicas, instalações físicas, equipamentos e recursos humanos adequados à prestação de assistência especializada de alta complexidade para o diagnóstico definitivo e tratamento dos cânceres mais recorrentes.

No ano de 2012, o Núcleo Hospitalar de Epidemiologia do Hospital de Base Ary Pinheiro, passou a ser a Unidade Hospitalar habilitada para efetuar a coleta de dados referentes ao diagnóstico, tratamento e evolução dos casos de neoplasias malignas nesta Instituição por intermédio dos Registros Hospitalares de Câncer (RHC). O Núcleo hospitalar de epidemiologia/NHE-HBAP, de 2012 a 2017, era o único responsável por inserir informações ao banco de dados de pacientes diagnosticados e tratados pelo SUS com câncer em Porto Velho.

De acordo com o diretor do Hospital do Câncer de Barretos unidade Porto Velho, a baixa disponibilidade de serviços de referência no norte e a grande quantidade de pacientes de Rondônia que iam fazer seu tratamento em Barretos (São Paulo) fizeram com que houvesse interesse em construir um hospital oncológico na Região Norte do país. O Hospital do Amor da Amazônia (nome dado a unidade do Barretos) foi construído com doações de iniciativa privada e inaugurado em 2017.

Em 2012 o serviço do hospital de Barretos, que já funcionava em uma pequena ala do Hospital de Base, era quem detinha os dados dos pacientes diagnosticados e tratados com câncer pelo hospital de Barretos (Barretinho. Todos os dados aqui analisados são de pacientes tratados no Hospital de Base e no Barretos/Barretinho (Hospital de Amor da Amazônia) entre os anos de 2012 a 2016. O Hospital de Base trabalha com diagnóstico e tratamento da doença, enquanto o Barreto/Barretinho atualmente trabalha com diagnóstico, tratamento e prevenção. No Hospital São Pelegrino é realizado o tratamento de quimioterapia.

As Figuras 2 e 3 representam a espacialização dos casos diagnosticados de câncer do colo do útero e mama feminino, pois para Santos, Brito \& Santos (2009) a utilização de 
mapas e imagens na análise da distribuição espacial de situações de risco e dos problemas de saúde, contribuem para a integração de dados demográficos, socioeconômicos e ambientais, promovendo o inter-relacionamento das informações de diversos bancos de dados, possibilitando estratégicas para efetivação das políticas públicas de saúde. O conhecimento do perfil epidemiológico do câncer de mama e útero em Porto Velho possibilita o melhor planejamento das ofertas de serviços, adequado às realidades inerentes a cada região, da prevenção ao acompanhamento de tratamento e reabilitação das pacientes.

As neoplasias malignas são frequentes no estado de Rondônia, entre os anos de 2012 a 2016, das 838 mulheres que fizeram exame, foram registrados 656 casos positivos de câncer de mama e útero. De acordo com a distribuição pelos 52 bairros que compõem a área urbana de Porto Velho, foram registrados 268 casos de câncer de colo de útero: 116 casos na zona leste da capital, a de maior incidência; na zona central ocorreram 76 casos; já na zona sul foram 59 casos e 17 na zona norte (Fig. 2).

Figura 2: Espacialização do número de casos de câncer de colo de útero por bairros de Porto Velho, RO - 2012-2016.

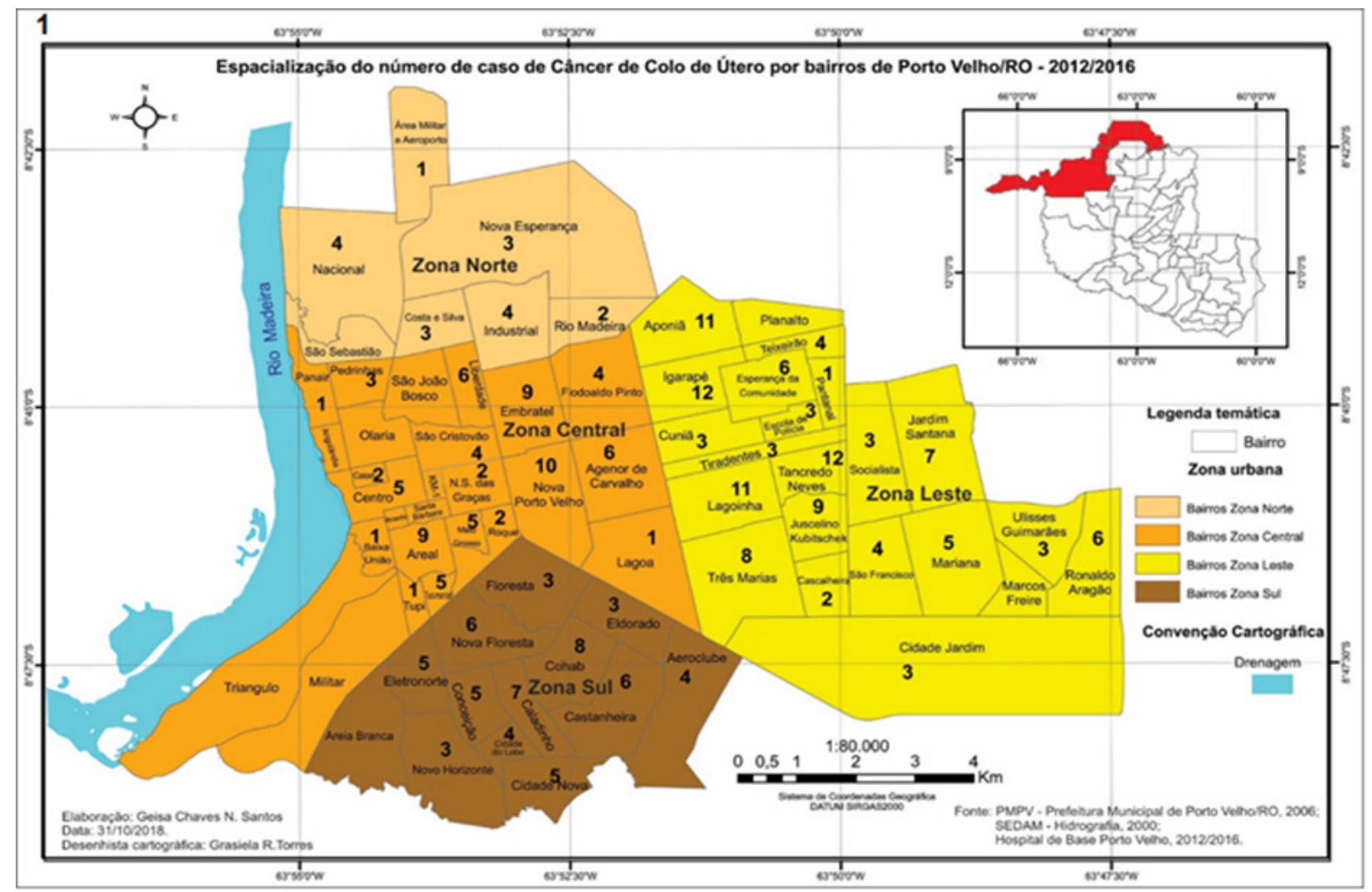

Já na Figura 3, a representação da ocorrência de câncer de mama de 388 diagnósticos ressalta a zona central com 154 casos; 116 na zona leste, 92 na zona sul e 26 casos na zona norte. 
Figura 3: Espacialização do número de casos de câncer de mama por bairros de Porto Velho, RO - 2012-2016.

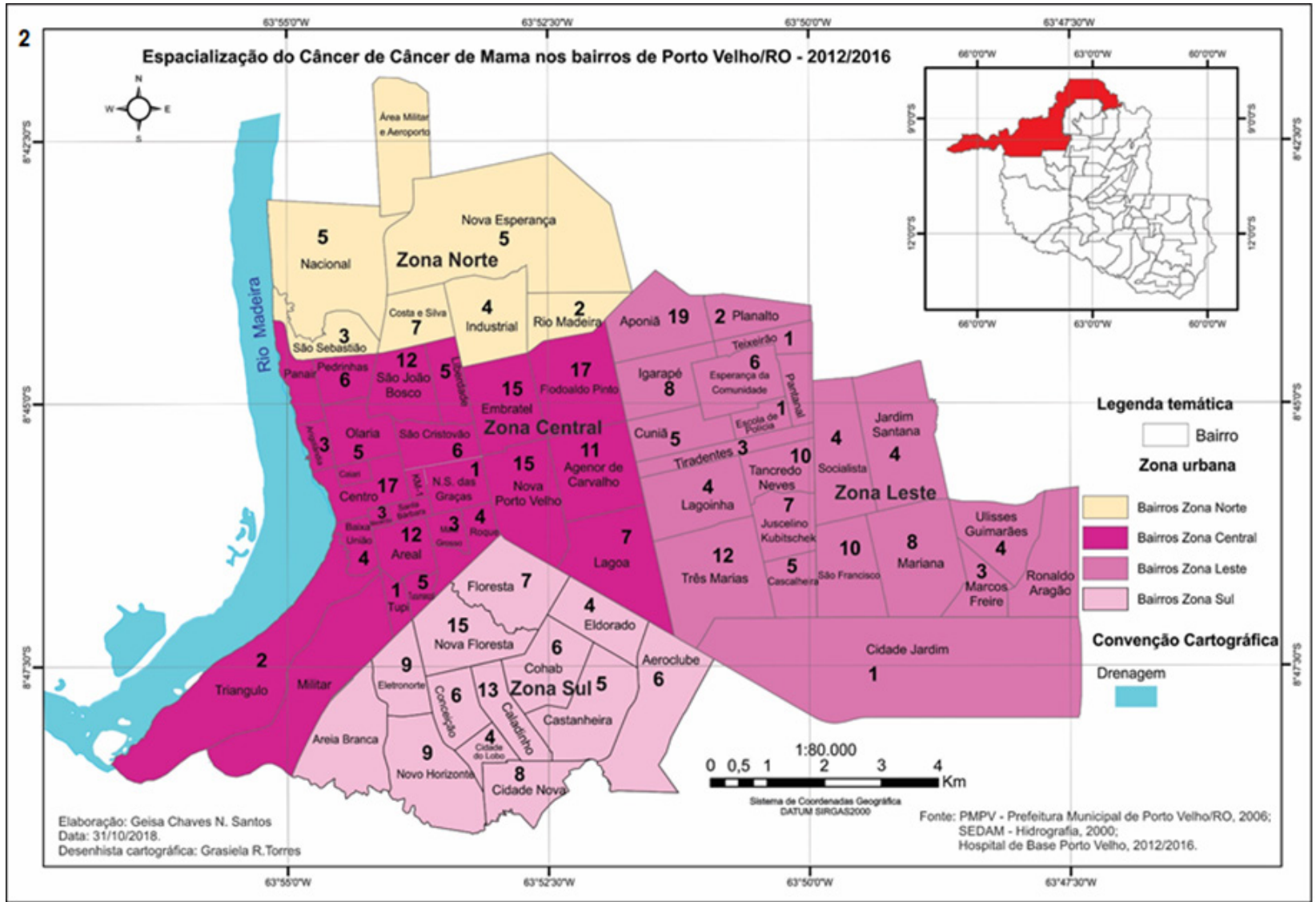

A respeito dos casos estudados, o hospital realiza o procedimento de questionário para anamnese das pacientes, com variáveis de raça/cor (sensu IBGE), idade, grau de escolaridade, estado civil e tabagismo.

Com relação à raça/cor (branca, parda, preta, amarela e sem informação), as duas neoplasias mostraram maior incidência em mulheres pardas, em todos os anos pesquisados (Fig. 4). 
Figura 4: Demonstrativo dos dados da anamnese de cor/raça dos cânceres de mama e de colo de útero em Porto Velho - 2012-2016.

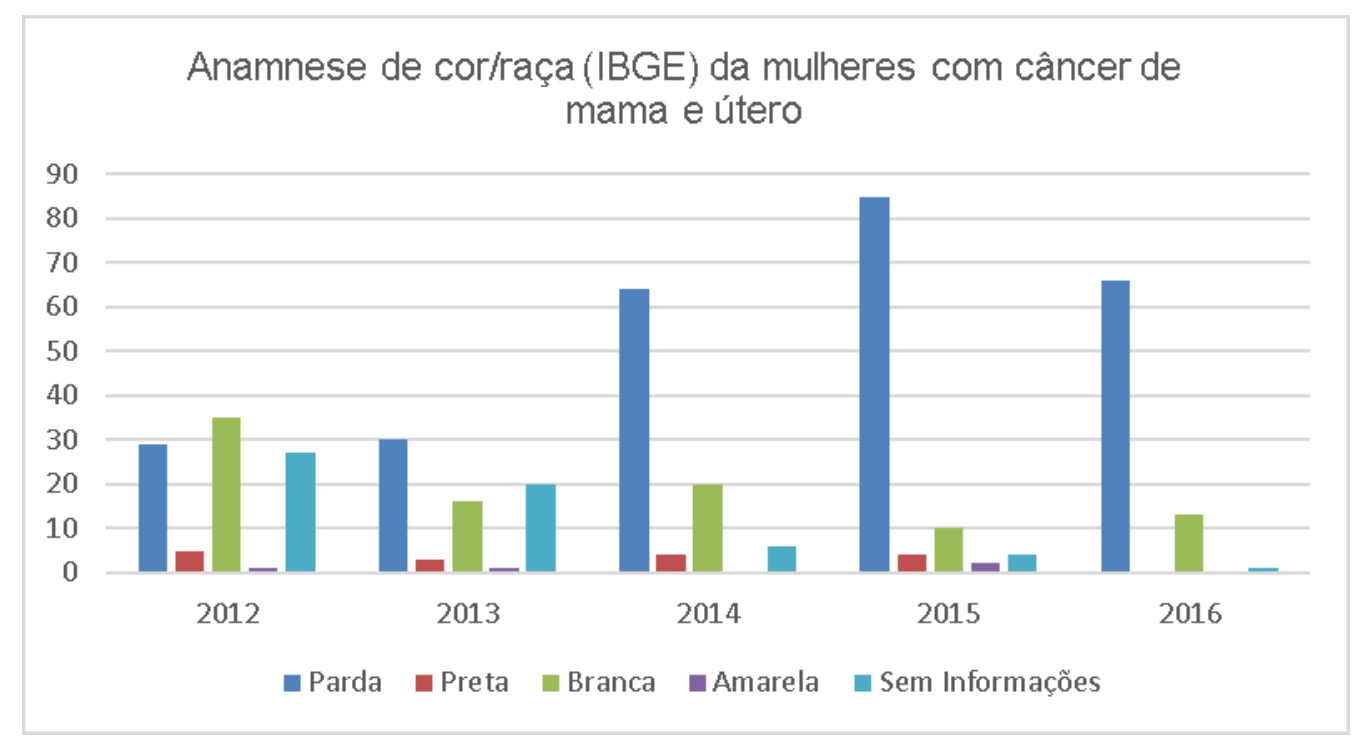

Em relação a idade, a maior prevalência de câncer de mama se deu na faixa etária de 41 a 70 anos (Fig. 5). Não houve diagnóstico positivo em mulheres entre 10 a 20 anos. No entanto, do total de 838 mulheres atendidas, 675 ficaram sem registro de idade demonstrando erro do atendente, dificultando qualquer inferência significativa sobre este dado.

Figura 5: Demonstrativo da anamnese da idade no câncer mamário em Porto Velho - 2012-2016.

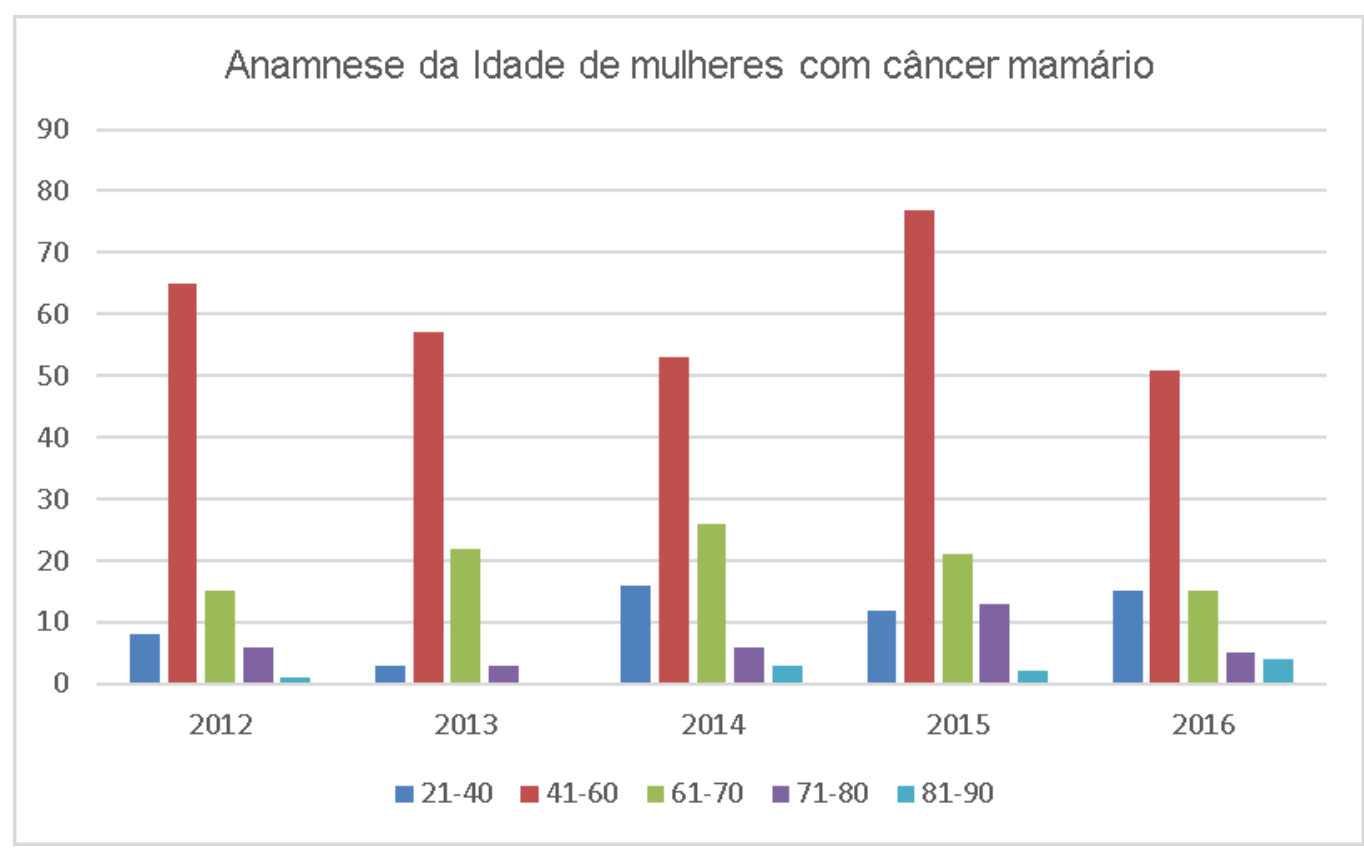

Ao tratar dos dados referentes ao câncer de útero foi evidenciado a prevalência desta doença em mulheres entre 10 e 20 anos de idade (Fig. 6). No entanto, deve ser lembrado, que o exame Papanicolau é mais comum do que a mamografia, e algumas vezes o auto exame de mamas não é realizado por jovens, portanto isso pode levar a ocorrer demora no diagnóstico da doença. 
Figura 6: Demonstrativo da anamnese de idade câncer uterino em Porto Velho - 2012-2016.

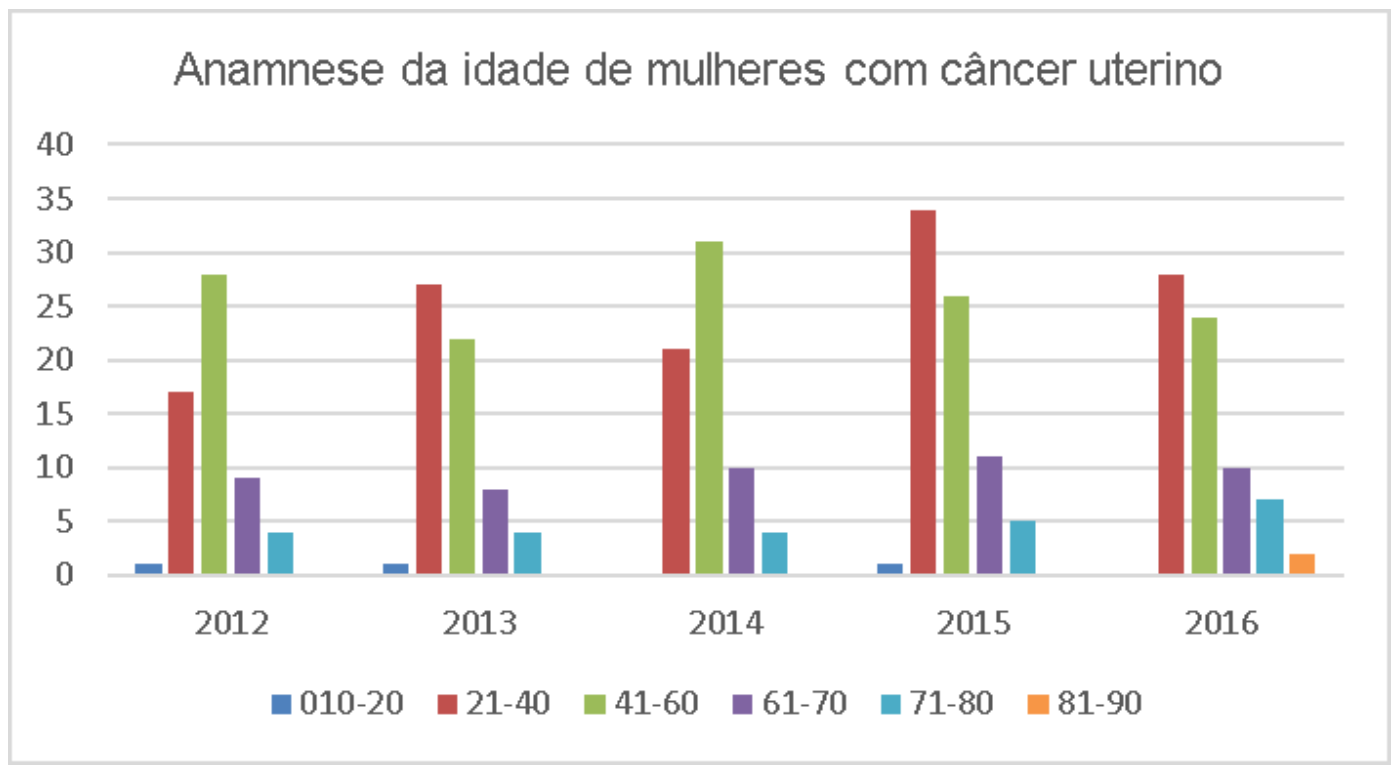

Com relação ao estado civil das pacientes (casada, solteira, separada judicialmente, união consensual, viúva e sem informação), pode ser visto na figura 7, que tanto o câncer de mama, quanto o de colo de útero incidem principalmente entre as casadas.

Figura 7: Demonstrativo da anamnese do estado civil de mulheres com câncer de mama e útero em Porto Velho - 2012-2016.

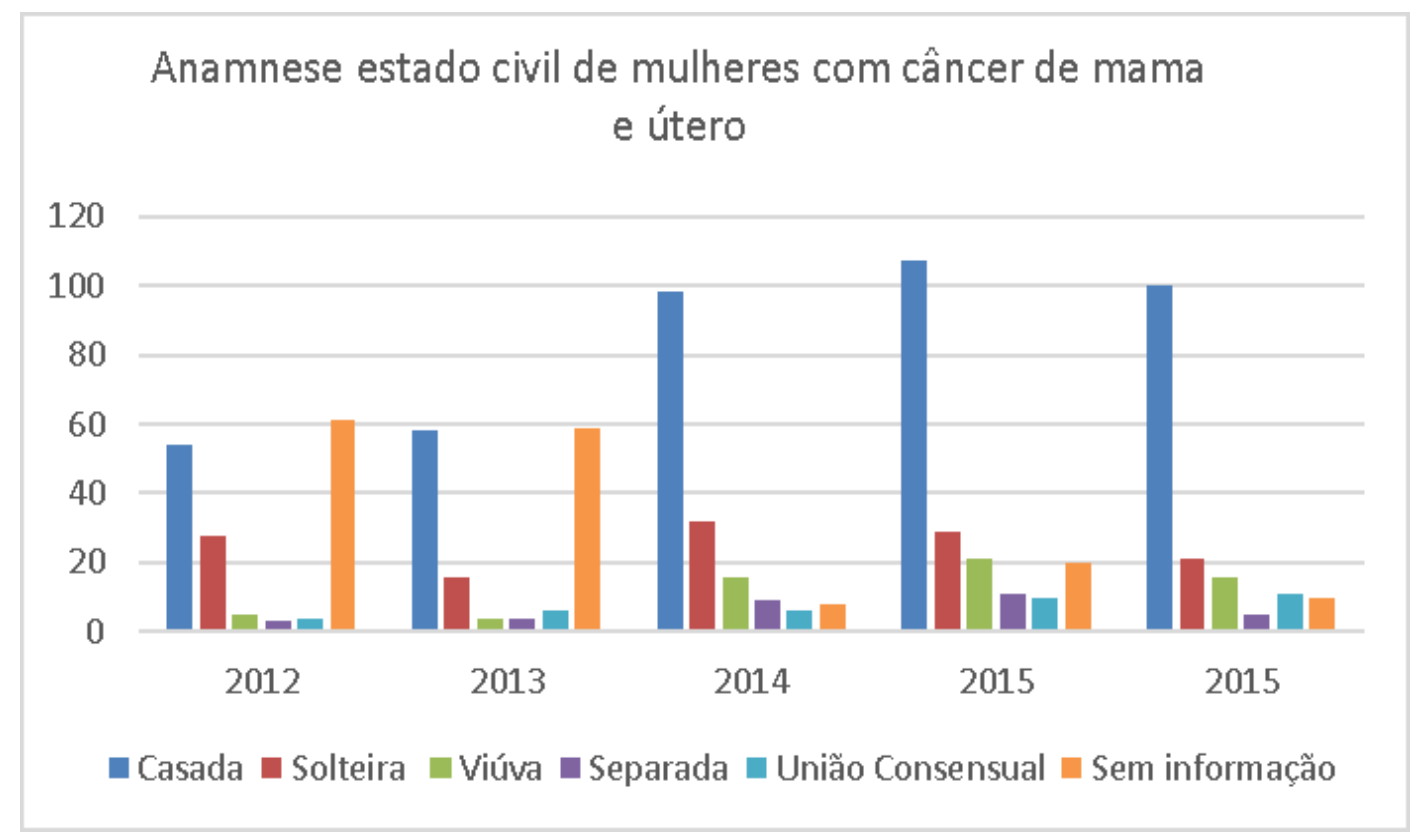

No que diz respeito a escolaridade (nenhuma, fundamental completo, fundamental incompleto, nível médio, nível superior completo, nível superior incompleto e sem informação), em ambas as neoplasias a maior incidência se deu sobre mulheres com o ensino fundamental incompleto (de mama) ou completo (de útero), conforme observado na figura 
8. Percebe-se que a falta de esclarecimento sobre o exame preventivo confere alto nível de risco às mulheres com menor grau de instrução.

Figura 8: Demonstrativo de anamnese referente a escolaridade nos casos de câncer mamário e uterino em Porto Velho - 2012-2016.

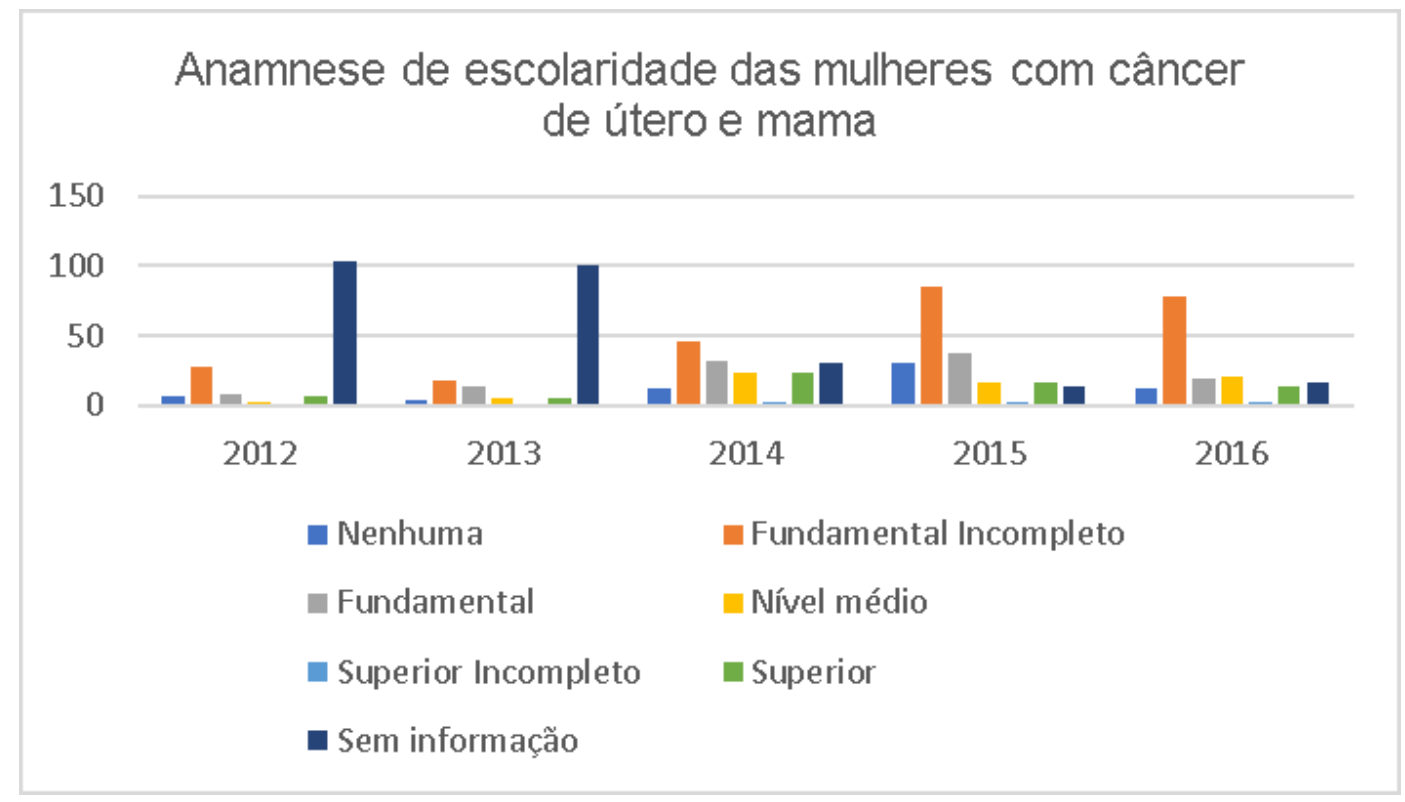

O tabagismo é um dos fatores que contribuem para alguns tipos de canceres no mundo, no entanto com relação a incidência de câncer de mama e útero não se percebeu valores significativos pois, 491 mulheres relataram nunca ter fumado, para 188 fumantes e 142 ex-fumantes.

De acordo com os resultados obtidos percebeu-se que o câncer de mama é mais frequente nos bairros da zona central da cidade, onde há maior número de mulheres que residem próximo ao seu local de trabalho, o setor comerciário. O que pode levar a inferir que a disponibilidade para os exames preventivos por parte dessas mulheres é menos frequente, pois não dispõem de tempo para ficar na fila de espera para marcar a consulta, depois outra fila para ser atendida, outra para marcar o exame, subsequente outra para fazer o exame e ainda mais uma para o retorno ao médico. Nesse sentido, há uma demanda de tempo que as trabalhadoras geralmente não possuem.

Já a espacialização do câncer de útero em Porto Velho se deu nos bairros da zona leste da cidade, onde há maior índice de mulheres com menos escolarização que desenvolvem atividades como domésticas, o que também as deixa o dia todo ocupadas, com pouca possibilidade de buscar por atendimento preventivo.

Portanto, o que há em comum entre as mulheres dessas duas zonas, heterogêneas no que tange ao acesso à infraestrutura, informação, rede de transporte, saneamento, é a falta de tempo das trabalhadoras para buscar por medidas preventivas do câncer, mamário ou de colo de útero. 


\section{DAS POLÍTICAS PÚBLICAS DIRECIONADAS AO TRATAMENTO DE CÂNCER DE MAMA E DE COLO}

São diversas as pesquisas direcionadas ao envelhecimento da sociedade e a mudança nos hábitos de vida, relacionados ao processo de urbanização, que fizeram com que a doença se tornasse mais presente e visível. Os governos nas instâncias federal, estadual e ou municipal realizam ações voltadas a conscientização, prevenção e cuidados, no entanto o câncer possui comportamento indeterminados, pois: ter muitos filhos pode causar câncer de colo do útero, não ter filhos pode causar câncer de mama - o que se tem certeza é que seu diagnóstico precoce, no Brasil, tem relação direta com o poder financeiro da paciente.

O direito a saúde está disposto na Constituição Federal de 1988 em seu artigo 196, e para isso organizaram-se as ações do Comitê Gestor da Política Nacional de Promoção da Saúde que, com a função de desenvolver ações direcionada a promoção da saúde em nível nacional, estadual e municipal, que reúne todas as secretarias do Ministério da Saúde, suas fundações, agências, Conselho Nacional de Secretários Municipais de Saúde (Conasems) e Conselho Nacional de Secretários Estaduais de Saúde (Conass). Coordena também a implantação da Política Nacional de Promoção da Saúde no SUS e em sua articulação com os demais setores governamentais e não-governamentais deve incentivar a elaboração, por parte dos estados, Distrito Federal e municípios, de programas, acolhimento, parcerias e campanhas para conscientização e tratamento da doença.

Na hierarquia dos poderes, fica a cargo do Governo Federal criar programas voltados para a saúde pública, com ações que visem a prevenção, diagnóstico, tratamento e reabilitação de pessoas. Já a responsabilidade pela prestação dos serviços de saúde se divide entre as esferas federal, estadual e municipal. O estado implanta projetos de governo, através de programas de ações voltadas para setores específicos.

O INCA é um órgão do Ministério da Saúde, integrante da Secretaria de Atenção à Saúde (SAS), criado pelo Decreto Presidencial 8065/2013. Também é o centro de Referência de Alta Complexidade em Oncologia do Ministério da Saúde, em conformidade com a Portaria MS 140/2014. O Instituto direciona sua atuação multidisciplinar ao desenvolvimento de programas e ações incluindo projetos, estudos pesquisas e experiências eficazes de gestão com instituições governamentais e não governamentais, além de manter acordos internacionais de cooperação em várias frentes formando redes de conhecimentos técnicos e científico e buscando diminuir o impacto da doença.

De acordo com INCA, são desenvolvidas ações tais como: campanhas e programas em âmbito nacional em atendimento à política nacional de prevenção e controle do câncer do Ministério da Saúde. Essas campanhas buscam o controle do câncer de colo do útero e controle do câncer de mama, são ações organizadas para reduzir a incidência, reduzir a exposição a fatores de riscos e a mortalidade pela doença e melhorar a qualidade de vida da mulher com câncer. Desde meados dos anos 1998 têm sido desenvolvidas políticas públicas por meio do Programa Viva Mulher (Brasil, 2002).

O controle do câncer de mama e colo do útero é hoje uma prioridade na agenda de saúde do país, e integra ações de saúde estratégicas para o Enfrentamento das Doenças 
Crônicas Não Transmissíveis (DCNT) lançada pelo Ministério da Saúde em 2011. A agenda estratégica elaborada pelo Estado é um conjunto de ações para o controle do câncer, com base em metas e resultados em áreas prioritárias do país direcionadas ao controle do câncer de colo do útero. Os objetivos são garantir o acesso ao exame preventivo de qualidade para as mulheres de 25 a 64 anos de idade e qualificar os diagnósticos e tratamento das lesões precursoras de câncer.

Já o Sistema de Informação do Câncer do Colo do Útero - SISCOLO, foi desenvolvido pelo INCA em 1999, em parceria com o Departamento de Informática do SUS (Datasus), como ferramenta de gerência das ações do programa de controle do câncer de colo do útero. Com este sistema é possível avaliar a população alvo, a qualidade dos exames, dentre outras informações com relevância para o acompanhamento da melhoria das ações de rastreamento diagnóstico e tratamento.

Em 2008 foi iniciado um projeto para apoiar a ações de capacitação profissional de médicos na atenção secundária à saúde, de forma regionalizada, possibilitando a implantação de serviços de referência para o diagnóstico e tratamento de lesões precursoras a nível ambulatorial. Desenvolveram-se os Centros Qualificadores de Ginecologistas para Assistência Secundária às Mulheres com Lesão Intraepitelial do Colo do Útero, e o aperfeiçoamento da gestão das ações de detecção precoce. Com o apoio técnico ao planejamento e à avaliação das ações de detecção precoce do câncer nos estados, também foi estipulada a produção de boletins informativos para acompanhamento dos indicadores, difusão de experiências e intercâmbio institucional para melhorar as ações de controle de câncer do colo de útero em mulheres.

Em 2009, foi iniciado o projeto de Gestão da Qualidade do Exame Citopatológico, com o objetivo de impulsionar o monitoramento interno e externo da qualidade dos laboratórios de citopatologia (MIQ e MEQ). As ações incluíram a avaliação das diretrizes e construção de modelo de monitoramento para o plano de trabalho dos estados, na realização de diagnóstico nos prestadores de serviços do SUS, acompanhamento e monitoramento das atividades. Com busca de melhores resultados, entre a realização do exame, o resultado e o começo do tratamento, foi também em 2009 iniciado a intensificação do rastreamento da doença na região norte, considera com alto índice de mortalidade naquele período. Em 2013 foi publicada a Portaria 3.388, que visou garantir a qualidade do exame citopatológicos.

Somente em 2014 com a Portaria 189 foram definidos os parâmetros e incentivos financeiros de custeio e de investimento para funcionamento de serviços que, integrados à rede de Atenção à Saúde, realizem o diagnóstico e tratamento das lesões precursoras através de colposcopia, biópsia e exérese da lesão (EZT).

Campanhas do Ministério Público visam orientar mulheres à prevenção primária de câncer de colo de útero, como usar camisinha nas relações sexuais fazer higienização íntima, e realizar o exame o preventivo regularmente a partir dos 25 anos ou após iniciar a vida sexual. Algumas ações preventivas são apresentadas em cartilhas, folders, cartazes e catálogos.

Segundo Brasil (2011), no ano de 2004 o Ministério da Saúde (MS) publicou o Controle do Câncer de Mama, documento que recomenda as seguintes ações para rastreamento de 
câncer de mama em mulheres assintomáticas: exame clínico das mamas a partir dos 40 anos; mamografia para mulheres entre 50 e 69 anos, com intervalo máximo de dois anos entre os exames; exame clínico das mamas e mamografia anual, a partir dos 35 anos, para mulheres do grupo de risco.

Deve-se dar ênfase no diagnóstico precoce, pois é um fator importante para que se alcance um tratamento eficiente. Para isso busca-se aprimorar a identificação dos sintomas desta enfermidade sendo os mais frequentes: o nódulo palpável, descarga papilar (identificada como a secreção das mamas, fora do ciclo grávido puerperal, que deve ser analisada criteriosamente sendo fundamental caracterizar se é espontânea, se proveniente de ducto único ou múltiplo, se tem aspecto cristalino, colostro-símile, sanguinolento, seroso ou coloração esverdeada/ amarelada).

A radiografia da mama, mais conhecida como mamografia, é o método de detecção precoce considerado mais eficiente. Após a detecção da doença utiliza-se de ultrassonografia para identificação de nódulo cístico ou sólido. Dessa maneira pode-se saber qual a abordagem a ser realizada. Nos casos de controle, é realizado a mastectomia (retirada das mamas), retirada do nódulo, como também pode ser realizado o controle radiológico. Cada tratamento deve seguir os critérios estabelecidos pelo médico responsavel pela paciente.

\section{CONSIDERAÇÕES}

Em meados dos séculos XX, as preocupações com o câncer na sociedade brasileira se intensificaram. $\mathrm{O}$ câncer se tornou uma presença frequente na vida cotidiana, na medida em que o conhecimento em torno da medicina oncológica se alargava, tornando a neoplasia em uma vigorosa preocupação social. Mas mesmo hoje, diante de todo suporte tecnológico existente para o tratamento e diagnósticos dos cânceres, os resultados nem sempre são positivos.

Porto Velho mostrou números altos de pessoas com câncer, tanto de mama quanto de útero, principalmente por falta de informação. E, quando se analisa sua distribuição por bairros, os índices mais altos estão nos bairros que concentram as mulheres trabalhadoras defrontadas com a escolha entre ganhar o salário para sua manutenção e a prevenção básica de sua saúde.

As concepções da Geografia da Saúde neste trabalho envolveram a análise não só das pessoas envolvidas direta ou indiretamente, como é o caso dos pacientes e dos atendentes, mas também das políticas públicas e saúde. Também foi evidente o descompromisso com o preenchimento dos dados de anamnese, dificultando as pesquisas em Geografia da Saúde.

\section{REFERÊNCIAS}

Barcellos, C., Sabroza, P.C., Peiter, P., \& Rojas, L.I. (2002). Organização espacial, saúde e qualidade de vida: A análise espacial e o uso de indicadores na avaliação de situações de saúde. Informe Epidemiológico do SUS, 11(3): 129-138. Recuperado de https://www.arca.fiocruz.br/bitstream/icict/713/2/BARCELLOS_ Analise \%20espacial\%20e\%20uso\%20de\%20indicadores_saude_2002.pdf 
Brasil. Ministério da Saúde. Secretária de Assistência à Saúde. Instituto Nacional do Câncer (2002). Viva Mulher - Câncer do colo do útero: informações técnico-gerenciais e ações desenvolvidas. Rio de Janeiro: INCA. Recuperado de http://bvsms.saude.gov.br/bvs/publicacoes/viva_mulher.pdf

Brasil. Ministério da Saúde. Secretaria de Atenção à Saúde. Departamento de Atenção Básica (2006). Controle dos cânceres do colo do útero e da mama. Brasília.

Brasil, Instituto Nacional de Câncer (2011). ABC do Câncer: abordagens básicas para o controle do câncer. Rio de Janeiro.

Carvalho, A. R. L. (1972). Tumores e Geografia Médica. In C.S. Lacaz, R.G. Baruzzi, \& W. Siqueira Junior, W. (Orgs.) Introdução à Geografia Médica do Brasil. (pp. 489-503). São Paulo: Edgard Blucher.

Engel, J., Kerr, J., Schlesinger, R.A., Sauer, H., \& Hölzel, D. (2004). Quality of life following breast-conserving therapy or mastectomy: Results of a 5-year prospective study. Breast Journal, 10(3):223-231.

Fonseca, R.M GS. (2007). Gênero e saúde da mulher: uma releitura do processo saúde doença das mulheres. In R.A.Q. Fernandes, \& N.Z. Narchi (Orgs.). Enfermagem e saúde da mulher. (pp. 30-61). Santana do Parnaíba: Manole.

Fundação Oswaldo Cruz (2018). Prevenção ao câncer do colo do útero. Recuperado de https:/ / portal.fiocruz. $\mathrm{br} /$ noticia/ prevencao-ao-cancer-do-colo-do-utero

Gallo, C.V., Guerra, M. R., \& Mendonça, G.A. (2005). Risco de câncer no Brasil: tendências e estudos epidemiológicos mais recentes. Revista Brasileira de Cancerologia, 51(3):227-234.

Ganz, P.A., Lorna, K., Stanton, A., Krupnik, J. L., Rowland, J. H., Meyerowitz, B. E. ... \& Belin, T.R. (2004). Quality of life at the end of primary treatment of breast cancer: First results from the moving beyond cancer randomized trial. Journal of the National Cancer Institute, 96(5):376-387.

Gomes, R., Skaba, M.M.V.F., \& Vieira, R.J.S. (2002). Reinventando a vida: proposta para uma abordagem sócio-antropológica do câncer de mama feminina. Caderno de Saúde Pública, 18(1):197-204.

Junqueira, R.D. (2009). Geografia Médica e Geografia da Saúde. Revista Brasileira de Geografia Médica e da Saúde Hygeia, 5(8):57-91. Recuperado de http:/ / www.seer.ufu.br/index.php/hygeia/article/view/16931

Lacaz, C.S., Baruzzi, R.G., \& Siqueira Junior, W. (1972). Introdução à Geografia Médica do Brasil. São Paulo: Ed. USP.

Lima C., \& Zeferino L. (2008). Prevenção do Câncer: Oncologia para a Graduação. São Paulo: Tecmed.

Lima, L.A., \& Michalski, A. (2016). Porto Velho, expressões de uma cidade centenária. In Confins [online]: n. 29, p. 1-14. Recuperado de https://journals.openedition.org/confins/11579.

Liu G., \& Robins H. (2006). A História Natural e a Biologia do Câncer: Manual de Oncologia clínica. São Paulo: Fundação Oncocentro de São Paulo.

Marsillac, J. de, \& Merched, N. (1954). Câncer e estatística. Revista Brasileira de Cancerologia, 10(12):40-65.

Moreno, M.S., Santos, M.L., \& Pereira, V. M. (2009). Exame de Papanicolau: Qualidade do esfregaço realizado por alunos de enfermagem. Revista Brasileira de Cancerologia, 55(1):19-25.

Peiter, P.C. (2005). Geografia da Saúde na faixa de fronteira continental do Brasil na passagem do milênio. (Tese de Doutorado em Geografia). Universidade Federal do Rio de Janeiro, UFRJ, Rio de Janeiro, RJ, Brasil.

Rojas, L.I. (1998). Geografía y salud: temas y perspectivas en América Latina. Cad. Saúde Pública, 14(4):701711.

Sodré, A. (1984). A frequência do câncer no Brasil. Revista Brasil. Med., 18(23):229-232.

Santos, M. (1998). A Natureza do Espaço: Técnica e Tempo, Razão e Emoção. São Paulo: Ed. USP.

Santos, M. (2006). O Espaço do cidadão. São Paulo: Ed.USP.

Santos, F.A.P.S., Brito, R.S., \& Santos, D.L.A. (2009). Exame de Papanicolau: qualidade do esfregaço realizado por alunos de enfermagem. Revista Brasileira de Cancerologia, 55(10):19-25. 
Silva, M.M., \& Silva, V. H. (2005). Envelhecimento: importante fator de risco para o câncer. Revista Arquivos Médicos ABC, 30(1): 11-18. Recuperado de https://www.portalnepas.org.br/amabc/issue/view/34

Teixeira, L.A. (2009). O câncer na mira da medicina brasileira. Revista Brasileira de História da Ciência, 2(1):104-117. Recuperado de http://www.historiadocancer.coc.fiocruz.br/index.php/pt-br/producaocientifica-lista/78-o-cancer-na-mira-da-medicina-brasileira-revista-brasileira-de-historia-da-ciencia.

Data de submissão: 19/fev./2019

Data de aceite: 14/abr./2020 